\title{
Technology features of using gums in a creation of gel bases
}

Nina Raichuk, Olena Podobiy

National University of Food technologies, Kyiv, Ukraine

\section{Keywords:}

Gelling

Xanthan

Gum

Guar

Locust bean

Rheology

\section{Article history:}

Received 12.05.2016

Received in revised

form 23.07.2016

Accepted 01.09.2016

\section{Corresponding \\ author:}

Olena Podobiy

E-mail:

e.podoby@yandex.ua

\section{Abstract}

Introduction. The aim of this research is the studying of technological, physical-chemical and rheological properties of the gel bases, based on hydrocolloids, for further development of gel food products.

Materials and methods. As objects of the research we have selected xanthan gum, guar gum and locust bean gum, also gel bases with different concentration of these gums. Samples were prepared, using 3 methods, to study the technological, physicalchemical and rheological properties of gel bases. The study of structural and mechanical properties of the samples were performed on the rotational viscometer.

Results and the discussion. It was investigated the influence of a gelling agent concentration, water temperature and the duration of swelling on the viscosity of colloidal solutions and on the limiting shift stress. Gel bases, prepared using 3 methods with chosen hydrocolloids, belong to structured systems. The analysis of the experimental data showed that the greatest value of the maximum viscosity that meets virtually indestructible system, for samples of gel bases with xanthan gum, prepared by the method 3, is $106,3 \mathrm{~Pa} \cdot \mathrm{s}$, with locust bean gum it is $1,97 \mathrm{~Pa} \cdot \mathrm{s}$, with guar gum it is $17,7 \mathrm{~Pa} \cdot \mathrm{s}$, at a concentration of $1 \%$.

Based on the experimental data it was also calculated the structural and mechanical characteristics. For the xanthan and guar gum the value tension of change is more than zero, this indicates that the investigated samples are structured bodies like solid. For the samples with locust bean gum the value tension of change is zero, this indicates that the investigated samples appear to be pseudo-plastic liquid. The most dynamic limit of ability to flow was observed in samples with xanthan gum, prepared by the second and the third methods, with locust bean gum and guar gum, prepared by the first and the third methods at a concentration of $1 \%$.

A comparison of hydrocolloids solutions, obtained at different ways of preparation, showed that the most appropriate preparation of gels is the third, because under these conditions the viscosity of solutions (at the same concentration of a gelling agent) was higher and the structure of the gel was more homogeneous.

Conclusions. All the experimental samples of gels are characterized by non-Newtonian pseudo-plastic type of stream. The most appropriate is the preparation of gels by the third method, which consisted in swelling of hydrocolloid for 40 minutes in water of room temperature, heating to $60{ }^{\circ} \mathrm{C}$ and cooling. The optimum concentration of gelling agents $-0.5 \%$. 


\section{Introduction}

Structured products have become widespread, they are easily digested and by allowing the inclusion various ingredients to them, they can extend the range of food products with high biological value. The creation of gel products is based on the concept of the formation of the suspension of vital substances that are ready for absorption by the organism, through being in a microenvironment of a gel. They are ready for consumption and quick assimilation always and anywhere [1].

It is known that in recent years it is a tendency to create products, based on natural substances, so studying the properties of xanthan gum, locust bean gum, guar gum is an urgent task [2]. The determining of the structural viscosity, thixotropic degree can objectively estimate the quality of a developed gel product.

The aim of this work is the studying of technological, physical-chemical and rheological properties of the gel bases, based on hydrocolloids, for further development of gel food products.

\section{Literature review}

The design and the implementation of new generation food occur mainly in two ways: the development of analogs of products, which quality criteria are the characteristics of known by population products, and the manufacturing of products with new properties and composition. The development of new forms of products, studying their consumer characteristics, quality and efficiency become more and more important[1-3].

As a result of years of research, scientists have concluded that the most effective way to deliver the necessary substances to the organism is a product in a gel form, which facilitates the biological digestion of products.

Functional products in the form of gels deliver equally well as hydrophilic and hydrophobic substances into the body. The number of active components is unlimited and typically gel products are multi-component composition [2]. Thickeners play a role not only of supporting components, but they are biologically active, having a beneficial effect on the human body.

Food stabilizers, thickeners and gelling agents are derived from many natural sources, including bacteria, terrestrial and marine plants and connecting tissues of animals. The possibility and feasibility of using these ingredients are determined by the totality of their properties, price and availability [3, 4].

The ingredients, which are used in the food industry, are received by the extraction from various natural materials and are brought in food to get a certain structure and the necessary stability, fluidity and consumer characteristics.

These additives include traditional materials such as starch and gelatin. The most spread structural polymer of terrestrial plants is the cellulose and gums and products from algae. Also in the food industry it is allowed the use of microbial polysaccharides, which are xanthan, gellan and polluan [3-5].

Hydrocolloids have a significant effect on the properties of food and cosmetic products with content from several $\mathrm{mg} / \mathrm{kg}$ to high concentrations. Constant attention of scientists to these substances is caused by the importance of hydrocolloids for food technologies. With the use of modern methods of research in recent years it has been established the structure of these polymers and the formation of mesh structures by them and mechanisms of their interaction with other polymers. These structures exhibit many properties of ready products, including the stability of emulsions, the lasting stability of suspensions $[2,6]$. 
"They are present in nearly all manufactured products of food and cosmetic industry, but at the same time they are not well investigated" [2]. This statement clearly shows the role of stabilizers, thickeners and gelling agents. It is impossible to enumerate all the functions of these additives, that have an influence on the appearance, "sensation in the mouth" and the taste of products not only because a lot of them, but also because of constant changes, because all the time there are new data of the use of vehicles and useful properties of these compounds.

All stabilizers, thickeners and gelling agents are united by common name hydrocolloids, have vegetable matter, some of them are received using biotechnology, and gelatin belongs to the products of animal origin .

One of the most important properties of hydrocolloids, that affects the texture, is their property to gelling. For example, the addition of even small amounts of carrageenan to jelly dairy desserts forms stable gels. Other classic gelling agents include pectin, gelatin and agar $[3,7]$.

One of the perspective gelling agents is gum. Gums are the natural polysaccharides; used for a manufacturing of food products as a stabilizer, thickener, gelling agent.

Xanthan gum (E415) is a natural polysaccharide, natural food additive that is used for food production as a stabilizer, thickener, gelling agent and means for encapsulation. Xanthan gum is obtained by fermentation of sugar syrup by cultures of Xanthomonas campestris. The substance is easily soluble in water, almost independent of mechanical influences, doesn't lose its properties in a wide range of $\mathrm{pH}$ and temperatures [8].

Locust bean gum (E410) is a plant polysaccharide, which is obtained by grinding the endosperm of plants Ceratonia siligua. This polysaccharide is spread in the food industry where it is widely used for thickening aqueous phase and prevent syneresis [9].

Guar gum (E412) is a powder white or yellowish color with a characteristic odor. It is extracted from grinded endosperm of guar beans. In food guar gum is used as a thickener, which has the following properties: regulates viscosity, stabilizes emulsions and provides creamy texture of products [10].

\section{Materials and methods}

As objects of the research we have selected xanthan gum, guar gum and locust bean gum, also gel bases with different concentration of these gums.

It was investigated three ways to study the technological conditions of preparation of gel bases.

By the way 1: to distilled water of room temperature with constant stirring we added hydrocolloid by parts and left for a day until the complete dissolution of a gelling agent. By the way 2: to distilled water, heated to $60{ }^{\circ} \mathrm{C}$, with constant stirring we added a gelling agent by parts to the formation of a gel. By the way 3: to purified water of room temperature we added the structure-made substance, heated to $60{ }^{\circ} \mathrm{C}$ and stirred to the formation of a gel. As the result we obtained transparent gel bases pale-white color, homogenous, various viscosities.

The study of structural and mechanical properties of the samples were performed on viscometer $[11,13]$. 


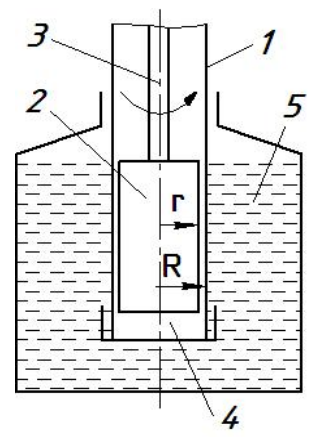

Figure 1. Device "Rheotest - 2"

Viscosity measurements were carried out using the device "Rheotest-2", which operates on a constant shear rate.

1. Prepare a sample of 100 cubic centimeters of the set system, mix her and maintain 20-30 minutes.

2. In the immobile external cylinder of device 1 (Figure 1) inundate $30-40 \mathrm{~cm}^{3}$ of the investigated system.

3. Put an internal cylinder 2 on an axis 3 that is connected with an electric engine.

4. An external cylinder 1 with the structured system is put on the fixed internal cylinder 2 and lift to support.

5. Fix position of external cylinder by means of nut. The investigated system is evenly distributed in a gap 4 between coaxial cylinders (external 1 and internal 2). If necessary the system is maintained in a thermostat 5 at a certain temperature (Figure 1)

6. On condition of permanent tension of change $\mathrm{P}=$ const to the internal cylinder deformations (12 or 24) give certain permanent speed, here an external cylinder stays still.

7. Register velocity of circulation of movable cylinder 2 by means of potentiometer. Velocity of circulation of cylinder is proportional to speed of deformation of the investigated system.

8. Tensions of change of $P$ expect, that arises up in the system, after equalization:

$$
P=z \times a
$$

$\mathrm{Z}$ - became internal cylinder (driven to the passport of device, for example, for the cylinder of $\mathrm{S} 2-\mathrm{Z}=5,39 \mathrm{~Pa}) ; a-$ it is a value of scale on an indicatory device (potentiometer).

9. The values of gradients of deformation $\varepsilon$ for every velocity of circulation (12 values) take from passport data.

10. After the values of tension of change of $P$ and gradient of deformation $\varepsilon$ expect dynamic viscosity $\eta$ :

$$
\eta=P / \varepsilon
$$

$\eta$ - dynamic viscosity, $\mathrm{Pa} \cdot \mathrm{s} ; \mathrm{P}$ - is tension of change, $\mathrm{Pa} ; \boldsymbol{\varepsilon}-$ it is speed of change, $\mathrm{s}^{-1}$.

The experimental data build complete rheological curves of viscosity $\eta=f(P)$ and fluidity $\dot{\varepsilon}=f(P)[11-13]$. 


\section{Results and discussion}

It was investigated the influence of a gelling agent concentration, water temperature and the duration of swelling on the viscosity of colloidal solutions and on the limiting shift stress.

It was calculated structural and mechanical properties of obtained colloidal systems.

For systems with xanthan gum, locust bean gum and guar gum with little stress we see an avalanche destruction of the structure, characterized by a sharp decrease of system viscosity. With further increase of the stress structure is destroyed more slowly, the viscosity decreases.

So, gel bases prepared by 3 ways with selected hydrocolloids belong to structured systems. Samples, prepared by the first method (Figure 1,4) for xanthan and locust bean gum didn't meet the required structural parameters.

The analysis of the experimental data showed that the greatest value of the maximum viscosity $\mathrm{y}_{0}$ that meets virtually indestructible system [13-15], for samples of gel bases with xanthan gum, prepared by the method 3 , is $106,3 \mathrm{~Pa} \cdot \mathrm{s}$ at a concentration of $1 \%$ (Figure 3 ). The lowest value of the minimum viscosity $\eta_{\mathrm{m}}$, corresponding to the almost destroyed system [13-15], is characteristic to the sample with xanthan gum, prepared by method 2, and it is $0,16 \mathrm{~Pa} \cdot \mathrm{s}$ at a concentration of $0.1 \%$ (Figure 2,3). The largest anomaly of the viscosity $\eta_{0}-\eta_{\mathrm{m}}$ is characteristic for the systems with xanthan gum, prepared by 3 method, and it is $105,3 \mathrm{~Pa} \cdot \mathrm{s}$ at a concentration of $1 \%$.

Based on the experimental data it was also calculated the structural and mechanical characteristics. For the xanthan gum the value $\mathrm{P}_{\mathrm{k} 1}>0$, this indicates that the investigated samples are structured bodies like solid [15-17]. The most dynamic limit of ability to flow was observed in samples with xanthan gum, prepared by the second and the third methods at a concentration of $1 \%$. An indicator $\mathrm{P}_{\mathrm{k} 1} / \mathrm{P}_{\mathrm{k} 2}$, which characterizes the strength of structural links in the system[15-17], indicates that samples prepared by the third method have the strongest structural links.

The analysis of the experimental data showed that the greatest value of the maximum viscosity $\mathrm{n}_{0}$ that meets virtually indestructible system [13-15], for samples of gel bases with locust bean gum, prepared by the method 3, is $1,97 \mathrm{~Pa} \cdot \mathrm{s}$ at a concentration of $1 \%$ (Figure6). The lowest value of the minimum viscosity $\eta_{\mathrm{m}}$, corresponding to the almost destroyed system[13-15], is characteristic to the sample with the locust bean gum, prepared by method 2, and it is $0,18 \mathrm{~Pa} \cdot \mathrm{s}$ at a concentration of $0.1 \%$ (Figure 5). The largest anomaly of the viscosity $\eta_{0}-\eta_{m}$ is characteristic for the systems with locust bean gum, prepared by the method 3, and it is $1,589 \mathrm{~Pa} \cdot \mathrm{s}$ at a concentration of $1 \%$.

Based on the experimental data it was also calculated the structural and mechanical characteristics. For the locust bean gum the value $\mathrm{P}_{\mathrm{k} 1}=0$, this indicates that the investigated samples appear to be pseudo-plastic liquid [15-17]. The most dynamic limit of ability to flow was observed in samples with locust bean gum, prepared by the first and the third methods at a concentration of $1 \%$. An indicator $\mathrm{P}_{\mathrm{k} 1} / \mathrm{P}_{\mathrm{k} 2}$, which characterizes the strength of structural links in the system [15-17], indicates that samples prepared by the third method have the strongest structural links.

The analysis of the experimental data showed that the greatest value of the maximum viscosity $\mathrm{y}_{0}$ that meets virtually indestructible system [13-15], for samples of gel bases with guar gum, prepared by the method 3 , is $17,7 \mathrm{~Pa} \cdot \mathrm{s}$ at a concentration of $1 \%$ (Figure9). The lowest value of the minimum viscosity $\mathrm{y}_{\mathrm{m}}$, corresponding to the almost destroyed system [13-15], is characteristic to the sample with the guar gum, prepared by method 2, and it is $0,43 \mathrm{~Pa} \cdot \mathrm{s}$ at a concentration of $0,1 \%$ (Figure 8 ). The largest anomaly of the viscosity $y_{0}-y_{m}$ is characteristic for the systems with guar gum, prepared by the method 3 , and it is $16,96 \mathrm{~Pa} \cdot \mathrm{s}$ at a concentration of $1 \%$. 


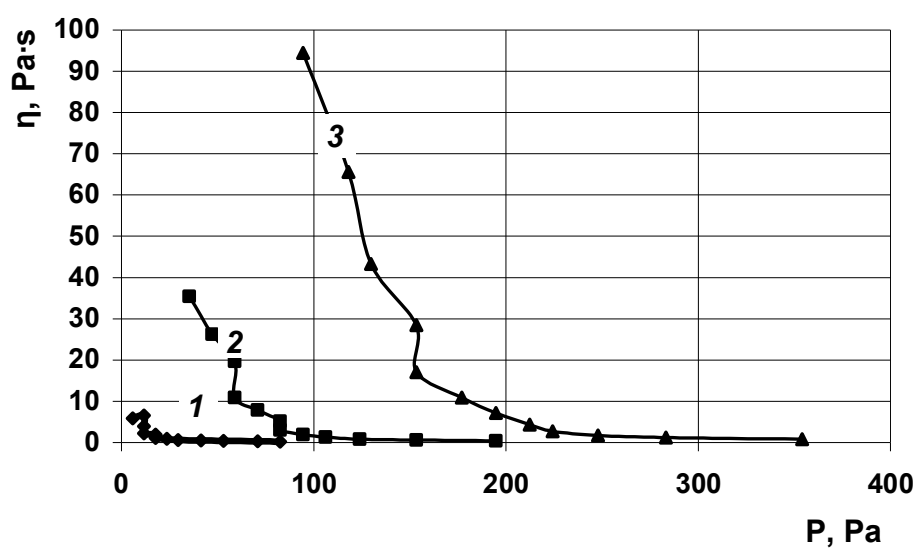

Figure 1. The viscosity curve of samples with xanthan gum, prepared by method 1

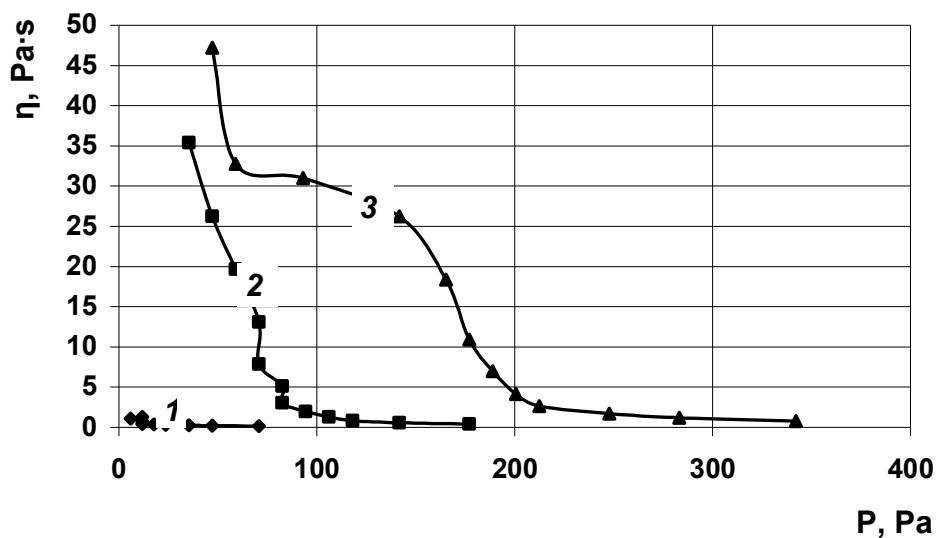

Figure 2. The viscosity curve of samples with xanthan gum, prepared by method 2

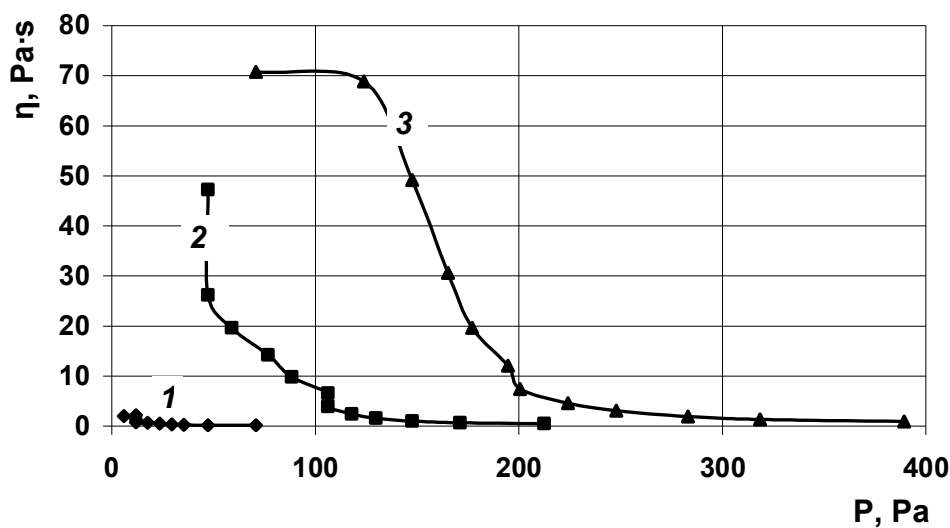

Figure 3. The viscosity curve of samples with xanthan gum, prepared by method 3

The concentration: $1-0,1 \% ; 2-0,5 \% ; 3-1,0 \%$ 


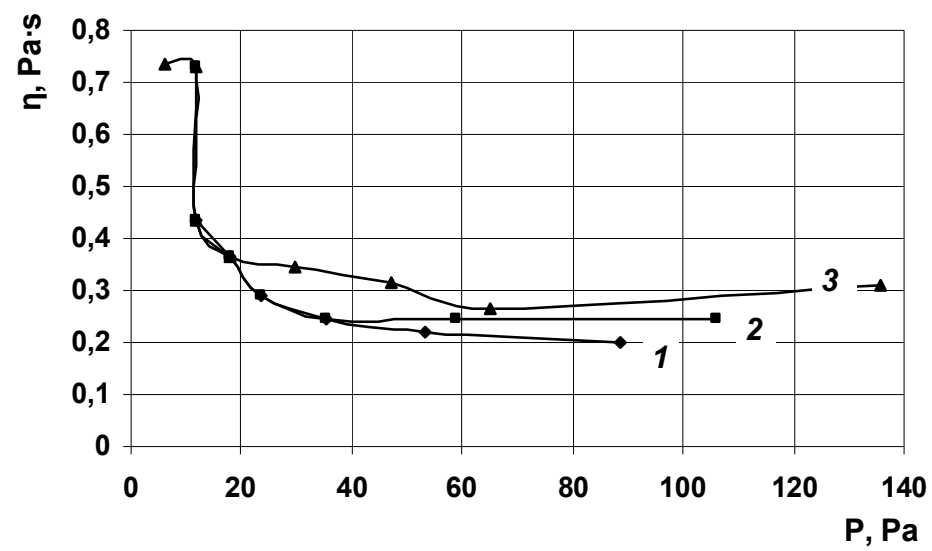

Figure 4. The viscosity curve of samples with loc. bean gum, prepared by method 1

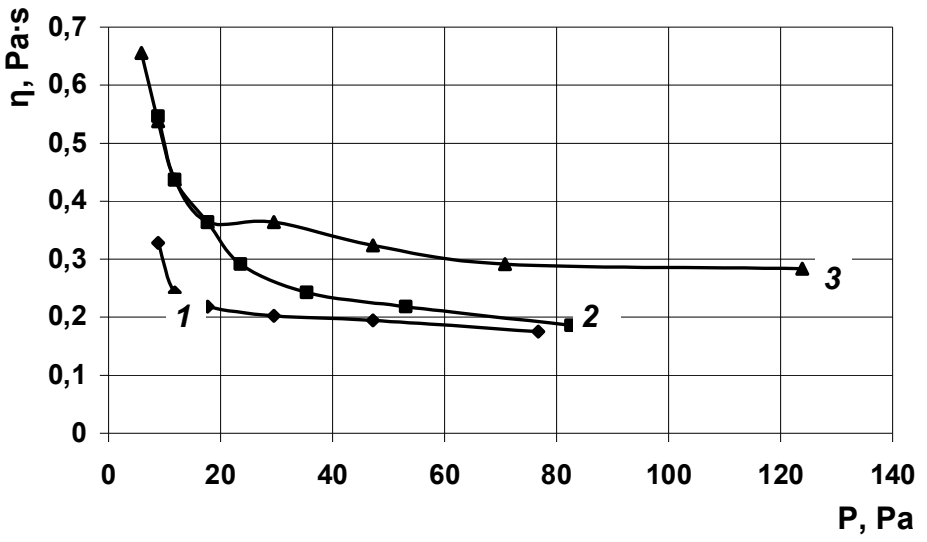

Figure 5. The viscosity curve of samples with loc. bean gum, prepared by method 2

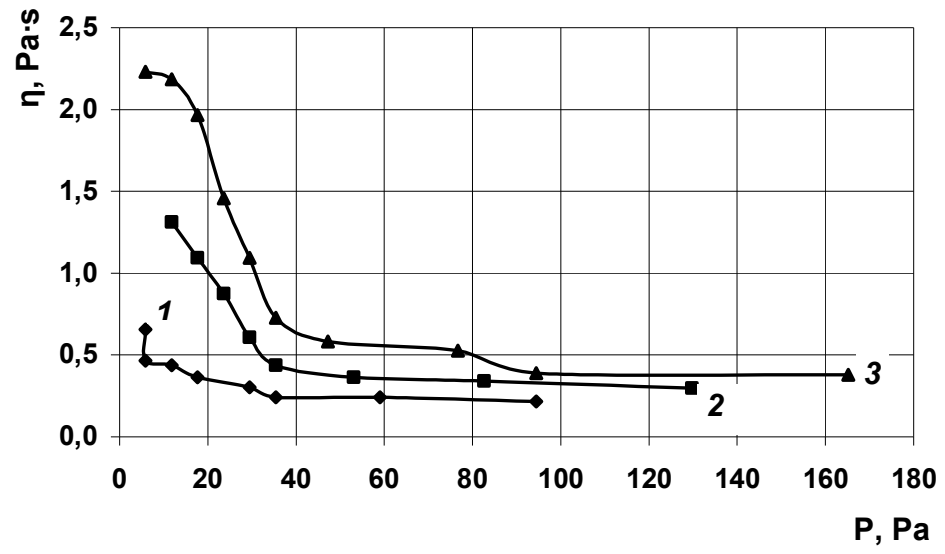

Figure 6. The viscosity curve of samples with loc. bean gum, prepared by method 3

The concentration: $1-0,1 \% ; 2-0,5 \% ; 3-1,0 \%$ 


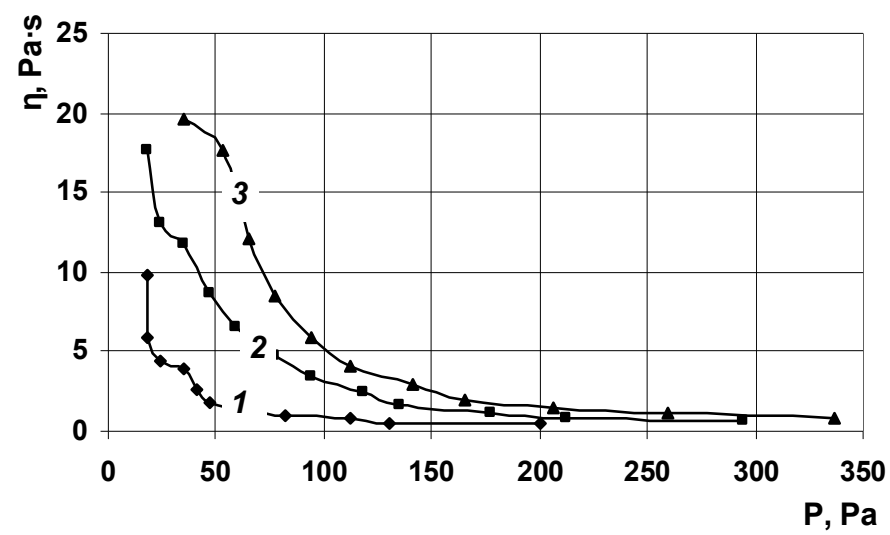

Figure 7. The viscosity curve of samples with guar gum, prepared by method 1

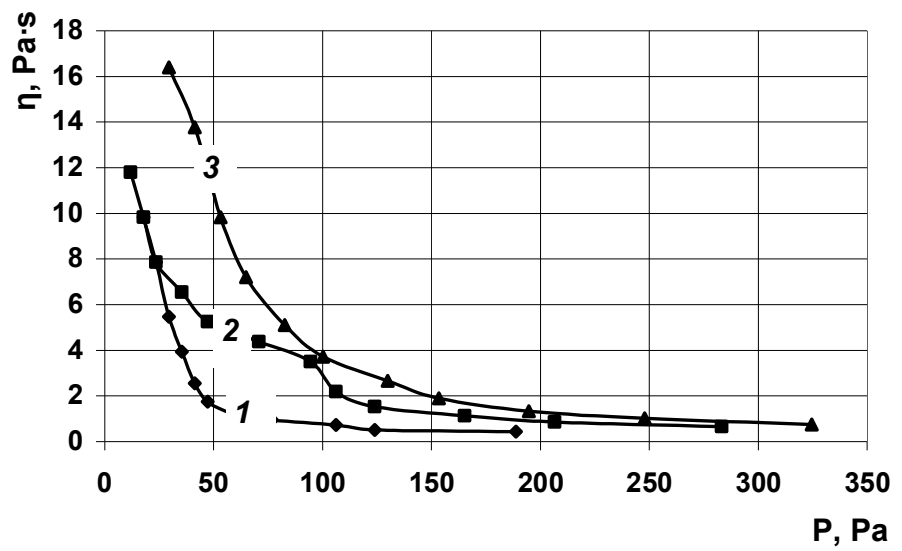

Figure 8. The viscosity curve of samples with guar gum, prepared by method 2

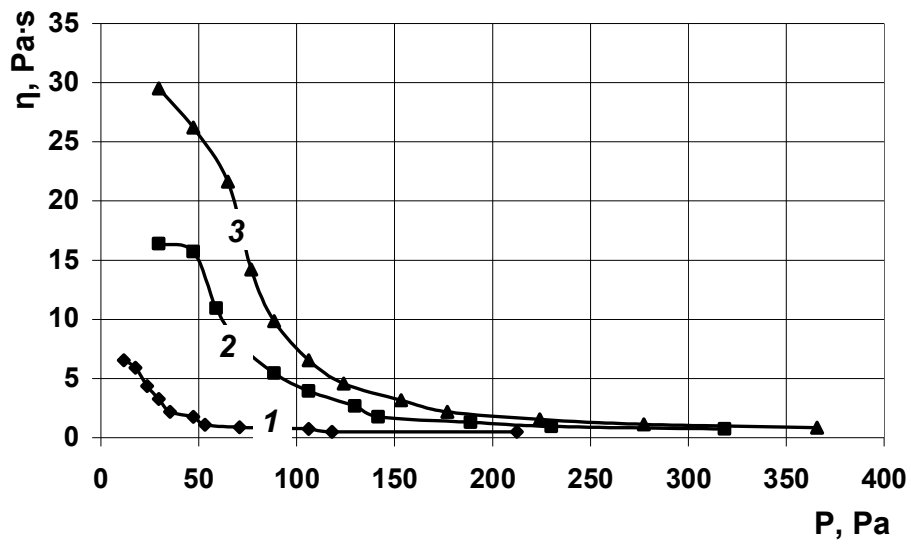

Figure 9. The viscosity curve of samples with guar gum, prepared by method 3

The concentration: $1-0,1 \% ; 2-0,5 \% ; 3-1,0 \%$ 
Based on the experimental data it was also calculated the structural and mechanical characteristics. For the guar gum the value $\mathrm{P}_{\mathrm{k} 1}>0$, this indicates that the investigated samples are structured bodies like solid [15-17]. The most dynamic limit of ability to flow was observed in samples with guar gum, prepared by the first and the third methods at a concentration of $1 \%$. An indicator $\mathrm{P}_{\mathrm{k} 1} / \mathrm{P}_{\mathrm{k} 2}$, which characterizes the strength of structural links in the system [15-17], indicates that samples prepared by the third method have the strongest structural links.

A comparison of hydrocolloids solutions, obtained at different ways of preparation, showed that the most appropriate preparation of gels is the third, because under these conditions the viscosity of solutions (at the same concentration of a gelling agent) was higher and the structure of the gel was more homogeneous. A process for preparing the hydrocolloid gels don't affect the fluidity of the systems.

\section{Conclusions}

All the experimental samples of gels are characterized by non-Newtonian pseudoplastic type of stream. In the study of dependence of the structural viscosity on the shift stress of samples it is found that the viscosity of xanthan gels, prepared by 3 ways, decreased with the increasing of shift stress. This dependence characterizes xanthan gels as structured dispersed systems.

The most appropriate is the preparation of gels by the third method, which consisted in swelling of hydrocolloid for 40 minutes in water of room temperature, heating to $60{ }^{\circ} \mathrm{C}$ and cooling. The viscosity of the samples, prepared by this way (with the same concentration of a gelling agent), was higher and the structure of the gel was homogeneous.

The optimum concentration of gelling agents, which ensures the effective structure of mudflow base, is $0,5 \%$. These gels have greater strength of structural links and greater range of stresses of structure destruction; also they meet the necessary organoleptic properties: have a more uniform structure, without lumps and do not stratify over time.

\section{References}

1. Singh J., Kaur L., Mccarthy O.J. (2007), Factors influencing the physico-chemical, morphological, thermal and rheological properties of some chemically modified starches for food applications, Food Hydrocolloid, 21, pp. 1-22.

2. Alan Imeson (2009), Food stabilisers. Thickeners and Gelling agents, pp. 15-50

3. Ahmt T.,Wischmann B., Blennow A., Madsen, F.,Bandsholm O., Thomsen J. (2004), Sensory and rheological properties of transgenically and chemically modified starch ingredients as evaluated in a food product model, Nahrung 48(2), pp. 149-155.

4. Piculell L., Bergfeld K., Nilsson S. (1995), Factors Determining Phase Behaviour of Multi-component Polymer Systems, Nottingham University Press, Nottingham, pp. $13-35$,

5. Aguilera J.M., Stanley D.W. (1999), Microstructural principles of food processing and engineering, Aspen Publishers, Gaithersburg, p. 432.

6. Achayuthakan P., Suphantharika M. (2008), Pasting and rheological properties of waxy corn starch as affected by guar gum and xanthan gum. Carbohyd, Polym., $71,9-17$

7. Ofner Clyde M., Klech-Geolotte Cathe M. (2002), Gels and jellies, Encucloprdia of Pharmaceutical Technology, pp. 1327-1344. 
8. García-Ochoa F. (2000), Xanthan gum: production, recovery, and properties, Biotechnol. Adv, 18(7), pp. 549-579.

9. Lozinsky V.I., Damshkaln L.G., Brown R., Norton I.T. (2000), Study of cryostructuring of polymer systems. On the nature of intermolecular links in the cruogels of ;locust bean gum, Polymer International, 49, pp. 1434-1443.

10. Dhugga K.S., Barreiro R., Whitten B., Stecca K., Tomes D. (2004), Guar seed synthase is a member of the cellulose synthase super gene family, Science, pp. 363-366.

11. Rosen Chochkov, Valentina Chonova, Stanimira Valova, Grozdan Karadzhov (2013), Rheological properties of fermented beverage from barley flour, Ukrainian Food Journal, 2(3), pp. 321-327

12. Popova A., Podobii O., Miroshnykov O., Stetcenko N. (2013), Research in rheological parameters of kefir, Scientific works of National University of food technologies, 48, pp. 132-138.

13. Rosalina I., Bhattacharya M. (2002), Dynamic rheological measurements and analysis of starch gels. Carbohyd. Polym., 48, pp. 191-202.

14. Tovar J.J., Melito C.C., Herrera E.E., Rascón A.A, Pérez E.E. (2002), Resistant starch formation does not parallel syneresis tendency in different starch gels, Food Chem., 76(4), pp. 455-459.

15. Kavanagh G.M., Ross-Murphy S.B., (1998), Rheological characterisation of polymer gels. Prog. Polym. Sci., 23, pp. 533-562.

16. Phillips G.O., Williams P.A., (2000), Handbook of Hydrocolloids, CRC Press, Washington, pp. 205-220.

17. Susan M.T., Alejandro G.M., Hallett F.R., Britt I.J. (2003), Aging dynamics in gelatin gel microstructure, Food Hydrocolloids, 17, pp. 503-513. 